\title{
BMJ Open Is length of time in a stroke unit associated with better outcomes for patients with stroke in Australia? An observational study
}

\author{
Doreen Busingye, ${ }^{1}$ Monique F Kilkenny, ${ }^{1,2}$ Tara Purvis, ${ }^{1}$ Joosup Kim, ${ }^{1,2}$ \\ Sandy Middleton, ${ }^{3}$ Bruce C V Campbell, ${ }^{4}$ Dominique A Cadilhac ${ }^{1,2}$
}

To cite: Busingye $D$, Kilkenny MF, Purvis T, et al. Is length of time in a stroke unit associated with better outcomes for patients with stroke in Australia? An observational study. BMJ Open 2018;8:e022536. doi:10.1136/ bmjopen-2018-022536

- Prepublication history and additional material for this paper are available online. To view these files, please visit the journal online (http://dx.doi org/10.1136/bmjopen-2018022536).

Received 22 February 2018 Revised 6 June 2018 Accepted 18 July 2018

\section{Check for updates}

(c) Author(s) (or their employer(s)) 2018. Re-use permitted under CC BY-NC. No commercial re-use. See rights and permissions. Published by BMJ.

For numbered affiliations see end of article.

\section{Correspondence to} Dr Dominique A Cadilhac; dominique.cadilhac@monash. edu

\section{ABSTRACT}

Objective Spending at least $90 \%$ of hospital admission in a stroke unit (SU) is a recommended indicator of receiving high-quality stroke care. However, whether this makes a difference to patient outcomes is unknown. We aimed to investigate outcomes and factors associated with patients with acute stroke spending at least $90 \%$ of their admission in an SU, compared with those having less time in the SU. Design Observational study using cross-sectional data. Setting Data from hospitals which participated in the 2015 Stroke Foundation National Audit: Acute Services (Australia) and had an SU. This audit includes an organisational survey and retrospective medical record audit of approximately 40 admissions from each hospital. Participants Patients admitted to an SU during their acute admission were included.

Outcome measures Hospital-based patient outcomes included length of stay, independence on discharge, severe complications and discharge destination. Patient, organisational and process indicators were included in multilevel logistic modelling to determine factors associated with spending at least $90 \%$ of their admission in an SU. Results Eighty-eight hospitals with an SU audited 2655 cases (median age 76 years, 55\% male). Patients who spent at least $90 \%$ of their admission in an SU experienced: a length of stay that was 2 days shorter (coefficient $-2.77,95 \% \mathrm{Cl}-3.45$ to -2.10 ), fewer severe complications (adjusted OR (aOR) $0.60,95 \% \mathrm{Cl} 0.43$ to 0.84 ) and were less often discharged to residential aged care (aOR $0.59,95 \% \mathrm{Cl} 0.38$ to 0.94 ) than those who had less time in the SU. Patients admitted to an SU within 3 hours of hospital arrival were three times more likely to spend at least $90 \%$ of their admission in an SU.

Conclusion Spending at least $90 \%$ of time in an SU is a valid measure of stroke care quality as it results in improved patient outcomes. Direct admission to SUs is warranted.

\section{INTRODUCTION}

Stroke remains a major global health challenge because it is a leading cause of death and major disability. ${ }^{1}$ It is well established that patients treated in stroke units (SUs) are more likely to receive evidence-based clinical practices, have better survival and self-rated

\section{Strengths and limitations of this study}

A strength of this research is that it has provided further evidence of the importance of length of time in a stroke unit, not just access, which has implications for clinical practice and development of new models of stroke care.

- The study involved a large comprehensive dataset, which provided national representation and used standardised data collection and an inclusive data dictionary to minimise reporting bias and ensure data were reliably collected.

- For some outcomes, only dates, rather than times were collected, which would have provided more accuracy.

- Design permits only association rather than determination of causality.

quality of life compared with those receiving care in other wards. ${ }^{2-4}$ Direct admission to the $\mathrm{SU}$ is recommended, preferably within 3 hours of stroke onset. ${ }^{5}$ Unless stroke is not a main clinical problem, guidelines also recommend that patients should be treated in an SU throughout their entire admission. ${ }^{6}$ Various factors can affect the time that patients spend in an SU. These factors include the bed capacity of the $\mathrm{SU},{ }^{7}$ bed management decisions, ${ }^{89}$ hospital policies, delays in the emergency department (ED), ${ }^{10}$ the clinical acuity of the patient whereby intubation or management in intensive care is warranted ${ }^{11}$ or delayed discharges for the next stage of care (eg, inpatient rehabilitation or aged care facility). Within Australia and in other counties, it has been recommended that 'spending at least $90 \%$ of the hospital admission in an SU' is an important indicator of high-quality acute stroke care. ${ }^{12-14}$ However, there is limited evidence that the proportion of time spent in the SU is associated with better outcomes in patients with stroke. In 
an observational study using data from the UK National Sentinel Audit of Stroke, lower case fatality was associated with spending more than $50 \%$ of hospital stay in the SU. ${ }^{15}$ Specific evidence is lacking relating to the benefits of spending $90 \%$ or more of the admission in an SU. In our study, we aimed to investigate in-hospital patient outcomes, and determine factors associated with patients with acute stroke spending at least $90 \%$ of their admission in an $\mathrm{SU}$, compared with those having less time in the SU.

\section{MATERIALS AND METHODS}

The description and reporting of this study is based on the Strengthening the Reporting of Observational Studies in Epidemiology statement. ${ }^{16}$

\section{Context of acute stroke care}

In Australia, the majority of patients with stroke are managed in public hospitals. It is usual practice that patients with suspected stroke or transient ischaemic attack present to the ED of hospitals, and are rapidly assessed, with brain imaging performed as a priority. Generally, all patients should be admitted to an acute $\mathrm{SU}$, or medical ward if the hospital has no available beds in the SU or does not have an SU or neurology ward. If patients require intubation or require higher acuity monitoring and one-to-one nursing care, they may also be managed in an intensive care unit. The median length of stay (LOS) in the acute setting is 5 days $(\mathrm{Q} 1,2$; Q3, $8),{ }^{17}$ after which, if rehabilitation is required, it is either provided in a separate subacute rehabilitation ward or hospital, or in a community setting.

\section{Study design and data source}

This observational study used data from hospitals participating in the Australian Stroke Foundation National Audit of Acute Services conducted in 2015. The audit programme is run biennially to provide cross-sectional data on clinical performance and has two components: an organisational survey and clinical audit. ${ }^{18}$ Detailed methods for the audit programme have been described elsewhere. ${ }^{18}$ In brief, data obtained in the organisational survey are used to describe aspects of acute stroke services, including bed numbers, admissions per year and available resources, for example, SUs. Data collected in the clinical audit are used to identify adherence to clinical guidelines and provide evidence on areas to improve the quality of care. Participation in the audit was voluntary and all Australian acute stroke services admitting at least three patients with acute stroke per year were eligible to participate in the organisational survey. Data for the first 40 or more consecutive acute stroke admissions (from 1 September 2014 and discharged by 28 February 2015) were collected by trained data abstractors from June to August 2015. To obtain a more representative sample, larger hospitals were encouraged to provide more cases. Patients with a primary diagnosis of acute stroke (ICD-10 codes: I61, I62.9, I63, I64) were eligible to be included in the audit.

\section{Patient population and definitions}

Data for patients who were treated at a hospital with an SU and only those patients admitted to an SU during their acute admission were included. Time spent in an SU (SU time) was determined by subtracting the date of discharge from the SU, from the date of admission to the SU. To determine patients who spent at least $90 \%$ of their admission in an SU, the SU time was divided by total LOS in the hospital (total LOS; calculated by subtracting date of discharge from hospital or death from date of admission to hospital). This corresponds to the admission to the respective acute care ward or commencement of an episode of care and the result multiplied by one hundred ([SU time/ total LOS]*100). We further determined early/late admission to the $\mathrm{SU}$ as $\leq 3$ hours versus $>3$ hours from arrival to the ED to admission on the SU. For patients whose stroke occurred while they were already in hospital, date of stroke onset was used as a surrogate for date of admission to hospital and arrival to ED.

The following patient outcomes were assessed: LOS, death, level of independence on discharge, severe complications and discharge destination. LOS was defined as the total length of time from admission to the hospital to discharge from the hospital or death. Level of independence on discharge was defined as a modified Rankin Scale (mRS) score of 0-2. A severe complication was a new event in hospital considered to be incapacitating, life threatening and one that prolonged hospital admission such as pneumonia, falls, fever, urinary tract infection, seizures and deep vein thrombosis. Discharge destinations included private residence, inpatient rehabilitation or residential aged care facility.

Only valid yes/no responses were included in the analyses for data related to medical history and the presence of symptoms on presentation to hospital. For data relating to processes of care, for example, received care in an SU, not documented and unknown responses were assumed to be negative and included in the denominator. To minimise bias, only patients with valid admission and discharge (SU and hospital) time or date were included.

\section{Statistical analysis}

Univariable analyses were performed to determine differences between patients who spent at least $90 \%$ and those who spent less than $90 \%$ of their admission in an SU. The $\chi^{2}$ test was used for categorical variables. The non-parametric Wilcoxon-Mann-Whitney rank-sum test was used for continuous variables that were not normally distributed.

Multilevel random effects logistic regression analyses, with level defined as hospital, were undertaken to determine:

1. The association between spending at least $90 \%$ of admission in an SU and in-hospital outcomes such as death, level of independence on discharge (mRS $0-2)$, severe complications and various discharge destinations. 
2. Factors associated with spending at least $90 \%$ of the admission in an SU.

For the continuous outcome of LOS, a median regression model with bootstrap estimated SEs was undertaken. A parsimonious approach to multivariable model development was used and independent variables with statistical significance $(p \leq 0.05)$ from univariable analyses were included.

To determine factors associated with spending at least $90 \%$ of the admission in an $\mathrm{SU}$, independent variables considered for inclusion in multivariable analyses were patient factors, for example, age; health system factors, for example, private hospital, presence of a stroke care coordinator and onsite neurosurgery and clinical process factors, for example, admission to SU within 3 hours of arrival to ED. Other potential confounders including stroke type (ischaemic vs intracerebral haemorrhage and unknown) and stroke severity factors such as inability to walk, arm weakness and speech impairment on admission and incontinence within 72 hours, which are based on the Counsell et al validated prognostic model for comparing patient outcomes ${ }^{19}$ were included. This validated mode ${ }^{19}$ has been compared against a model using age plus scores on the National Institutes of Health Stroke Scale and both prognostic models performed well overall, thus, the choice between them should be based on clinical and practical considerations. ${ }^{20}$

Models for association between length of time spent in an SU and in-hospital outcomes were adjusted for patient characteristics (eg, premorbid function and history of atrial fibrillation), variables with clinical importance (eg, sex and age), stroke type and stroke severity factors. Additional sensitivity analyses were undertaken, including:

i. Propensity score matching with stratification to minimise potential confounding by indication and compare between similar subgroups of patients (see online supplementary methods).

ii. Other cut-offs for percentage of admission spent in an SU (eg, $\geq 50$ to $<60, \geq 60$ to $<70, \geq 70$ to $<80, \geq 80$ to $<90$ ) were undertaken to determine a potential dose effect with LOS, severe complications and independence on discharge.

Standard techniques were implemented to check for collinearity. Values of $\mathrm{p}<0.05$ were considered significant for all analyses. Adjusted OR (aOR) or coefficients with 95\% CIs were calculated. Stata V.12.0 (Stata) statistical software was used for all analyses.

\section{Patient and public involvement}

Patients and/or the public were not involved in the development of this research project.

\section{RESULTS}

Overall, the clinical audit comprised data from 4087 patients at 112 hospitals. Most were public hospitals $(\mathrm{n}=104,93 \%)$ and were located in metropolitan areas $(\mathrm{n}=105,94 \%)$. Twenty-four of these hospitals $(\mathrm{n}=664$ patients) did not have an SU. Of the patients admitted to a hospital with an SU, 20\% ( $n=684)$ were not treated in an SU at any time during their admission. There were 2739 patients treated in an SU at some time during their admission. Eighty-four patients with invalid or missing dates of admission or discharge from the hospital or $\mathrm{SU}$ were excluded from the analyses. Overall, 2655 patients were assessed, whereby almost two-thirds (64\%) spent at least $90 \%$ of their admission in an SU. Compared with patients not treated on the $\mathrm{SU}$, patients admitted in an SU were more likely to be younger, male, independent prior to stroke and have an ischaemic stroke (online supplementary table A).

\section{Patient characteristics and clinical processes}

The median age for all included patients $(n=2655)$ was 76 years (Q1:65, Q3:84) and 55\% were male (table 1). Patients who spent at least $90 \%$ of their admission in an SU were more likely to be younger, and have less severe strokes, that is, fewer were unable to walk on admission or incontinent within 72 hours of admission compared with those who spent less than $90 \%$ of their admission in an SU (table 1 and online supplementary table B).

Importantly, patients who spent at least $90 \%$ of their admission in an SU compared with those who did not, were more likely to be admitted to an SU within 3 hours of arrival to ED, have a brain scan within 24 hours, be discharged from the hospital on the same day they were discharged from the SU (table 1), be assessed for rehabilitation by a physiotherapist within 48 hours of admission and have rehabilitation therapy commenced within 48 hours of their initial assessment (online supplementary table $\mathrm{C}$ ).

Patients who spent at least $90 \%$ of their admission in an SU had a shorter median time (hours) from arrival to the ED to admission on an SU compared with those who spent less than $90 \%$ of their admission in an SU (median time 6 hours, Q1: 4, Q3: 10 vs median time 17 hours, Q1: 6, Q3: 35; $\mathrm{p}=<0.001$ ).

\section{In-hospital outcomes and complications}

Complications, such as aspiration pneumonia, fever, urinary tract infections, falls, stroke progression and seizures, were less common in patients who spent at least $90 \%$ of their admission in an SU compared with those who spent less time in an SU (figure 1).

The median LOS (days) in the hospital for patients who spent at least $90 \%$ of their admission in an SU was significantly shorter than those who spent less than $90 \%$ of their admission in an SU (median LOS 4, Q1: 3, Q3: 8 vs median LOS 7, Q1: 4, Q3: 13; $\mathrm{p}=<0.001$ ). Patients who spent at least $90 \%$ of their admission in an SU were more likely to be independent on discharge and less likely to have any severe complication or die in the hospital (table 1).

On adjustment for confounding variables, no differences were detected in independence at discharge or death between the two groups (table 2). However, patients who spent at least $90 \%$ of their admission in an SU were 0.60 times less likely to have any severe complication and 
Table 1 Characteristics of patients with stroke who spent at least $90 \%$ and those who spent less than $90 \%$ of admission in an SU

\begin{tabular}{|c|c|c|c|}
\hline Spent at least $90 \%$ of admission in an SU & $\begin{array}{l}\text { Yes }(n=1687) \\
n(\%)\end{array}$ & $\begin{array}{l}\text { No }(n=968) \\
n(\%)\end{array}$ & $P$ values \\
\hline \multicolumn{4}{|l|}{ Patient characteristics } \\
\hline Age, median $(\mathrm{Q} 1, \mathrm{Q} 3)^{\star}$ & $75(65,84)$ & $77(66,85)$ & 0.006 \\
\hline Male & $936(55)$ & $537(55)$ & 0.99 \\
\hline Independent prior to stroke (mRS 0-2) & $1401(83)$ & $810(84)$ & 0.68 \\
\hline In-hospital stroke & $26(2)$ & $37(4)$ & $<0.001$ \\
\hline \multicolumn{4}{|l|}{ Stroke type } \\
\hline Ischaemic stroke & $1426(85)$ & $805(83)$ & 0.36 \\
\hline Haemorrhagic stroke & $162(10)$ & $114(12)$ & 0.08 \\
\hline Unknown stroke type & $99(6)$ & $49(5)$ & 0.38 \\
\hline \multicolumn{4}{|l|}{ Stroke severity $\dagger$} \\
\hline Arm weakness on admission & $1030(62)$ & $592(63)$ & 0.82 \\
\hline Impaired speech on admission & $987(60)$ & $554(59)$ & 0.52 \\
\hline Unable to walk on admission & $862(52)$ & $543(57)$ & 0.005 \\
\hline Incontinence at 72 hours of admission & $488(30)$ & $340(36)$ & 0.001 \\
\hline \multicolumn{4}{|l|}{ History of comorbidities } \\
\hline Atrial fibrillation $\ddagger$ & $418(28)$ & $276(33)$ & 0.01 \\
\hline Ischaemic heart diseaseł & $396(27)$ & $254(30)$ & 0.05 \\
\hline Previous stroke or TIA§ & $513(34)$ & $277(32)$ & 0.49 \\
\hline \multicolumn{4}{|l|}{ Clinical processes of care } \\
\hline Transferred to SU within 3 hours of ED arrivalł & $229(16)$ & $52(6)$ & $<0.001$ \\
\hline Transferred to SU within 24 hours of ED arrivalł & $1406(95)$ & $516(62)$ & $<0.001$ \\
\hline Brain scan within 24 hours of ED arrivalף & $1329(97)$ & $722(95)$ & 0.01 \\
\hline $\begin{array}{l}\text { Date of discharge from SU same as date of discharge from } \\
\text { hospital }\end{array}$ & $1567(99)$ & $456(52)$ & $<0.001$ \\
\hline \multicolumn{4}{|l|}{ Organisational characteristics } \\
\hline Metropolitan hospital & $1634(97)$ & 955 (99) & 0.004 \\
\hline Private hospital & $116(7)$ & $94(10)$ & 0.01 \\
\hline Stroke care coordinator present & $1030(61)$ & $550(57)$ & 0.03 \\
\hline Access to onsite neurosurgery & $566(34)$ & $402(42)$ & $<0.001$ \\
\hline Stroke team involved in quality improvement in last 2 years & $1507(89)$ & $831(86)$ & 0.008 \\
\hline Access to early supported dischargeteam & $229(14)$ & $102(11)$ & 0.02 \\
\hline Regular multidisciplinary team meetings & $1659(98)$ & $941(97)$ & 0.05 \\
\hline \multicolumn{4}{|l|}{ Number of beds on SU } \\
\hline$<5$ & $752(45)$ & $464(48)$ & 0.001 \\
\hline $5-9$ & $462(27)$ & $307(32)$ & \\
\hline$\geq 10$ & $473(28)$ & $197(20)$ & \\
\hline Stroke admissions last year $\geq 100$ & $1563(93)$ & $916(95)$ & 0.05 \\
\hline $\begin{array}{l}\text { Stroke specialist research nurse involved with } \\
\text { treatment }\end{array}$ & $319(19)$ & $140(14)$ & 0.004 \\
\hline Access to ongoing inpatient rehabilitation & $1554(92)$ & $916(95)$ & 0.01 \\
\hline \multicolumn{4}{|l|}{ In-hospital outcomes } \\
\hline Any severe complication ${ }^{* *}$ & $133(8)$ & $129(14)$ & $<0.001$ \\
\hline Independent on discharge (mRS 0-2) & $845(54)$ & $408(47)$ & 0.002 \\
\hline Died in hospital & $107(6)$ & $95(10)$ & 0.001 \\
\hline
\end{tabular}


Table 1 Continued

\begin{tabular}{|c|c|c|c|}
\hline Spent at least $90 \%$ of admission in an SU & $\begin{array}{l}\text { Yes }(n=1687) \\
n(\%)\end{array}$ & $\begin{array}{l}\text { No }(n=968) \\
n(\%)\end{array}$ & $P$ values \\
\hline \multicolumn{4}{|l|}{ Discharge destination (survivors) } \\
\hline Residential aged care facility & $74(5)$ & $77(9)$ & $<0.001$ \\
\hline Inpatient rehabilitation & $487(31)$ & $268(31)$ & 0.95 \\
\hline
\end{tabular}

${ }^{*}<1 \%$ unknown/not documented data.

†1\%-5\% unknown/not documented data.

$\ddagger 11 \%-15 \%$ unknown/not documented data.

$\S 6 \%-10 \%$ unknown/not documented data.

१16\%-20\% unknown/not documented data.

${ }^{\star \star} A$ complication considered incapacitating, life threatening and one that prolongs hospital admission, for example, pneumonia, falls, fever, urinary tract infection, seizures, deep vein thrombosis.

ED, emergency department; mRS, modified Rankin Scale; SU, stroke unit; TIA, transient ischaemic attack.

0.59 times less likely to be discharged to a residential aged care facility than those who spent less than $90 \%$ of admission in an SU (table 2). Patients discharged to aged care were more likely to be transferred from the SU to another ward/unit before being discharged from hospital regardless of how long they spent in the SU (Discharged aged care: $60 \%$ were discharged from the SU the same day as from hospital; other destination: $84 \%$ ).

Median LOS for patients who spent at least $90 \%$ of their admission in an SU was 2 days shorter than for those who did not. No difference in median LOS between groups for those patients who suffered a severe complication was evident (table 2). Results from the sensitivity analyses using propensity score matching provided evidence of benefit from a greater proportion of time spent in an SU when confounding by indication is controlled (online supplementary tables D and E). These results are consistent with our findings from the primary analysis.

Sensitivity analyses, including other cut-offs for percentage of admission spent in an SU (eg, $\geq 50$ to $<60$, $\geq 60$ to $<70$, $\geq 70$ to $<80, \geq 80$ to $<90$ ), provided evidence of a potential dose effect between occurrence of any severe complications and percentage of admission spent in an SU. In these analyses, in comparison to other cut-offs of percentage of admission

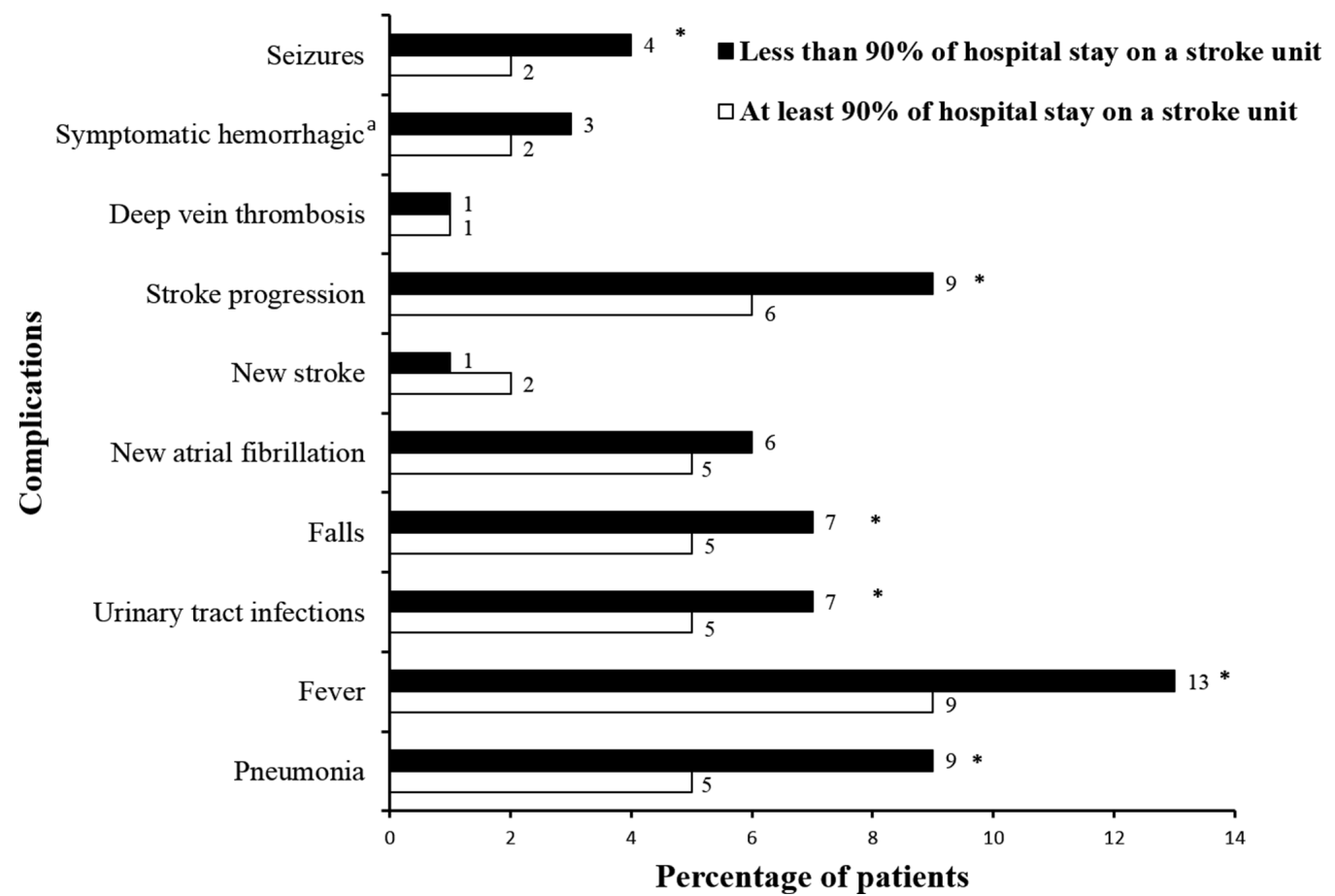

Figure 1 Differences in complications between patients who spent at least $90 \%$ and those who spent less than $90 \%$ of their admission in a stroke unit. *Significant $p<0.05$; ${ }^{a}$ symptomatic haemorrhagic transformation. 


\begin{tabular}{|c|c|c|c|c|}
\hline Model & Outcome & $\mathrm{aOR}^{*}$ & $95 \% \mathrm{Cl}$ & P values \\
\hline 1. & Any severe complication $†$ & 0.60 & 0.43 to 0.84 & 0.003 \\
\hline 2. & Independent on discharge (mRS 0-2) & 1.19 & 0.92 to 1.53 & 0.19 \\
\hline 3. & Died & 0.72 & 0.49 to 1.06 & 0.09 \\
\hline 4. & Discharged to private residence & 1.05 & 0.84 to 1.32 & 0.67 \\
\hline 5. & Discharged to inpatient rehabilitation & 0.97 & 0.76 to 1.23 & 0.79 \\
\hline \multirow[t]{2}{*}{6.} & Discharged to residential aged care facility & 0.59 & 0.38 to 0.94 & 0.03 \\
\hline & & Coefficient ${ }^{*}$ & $95 \% \mathrm{Cl}$ & $\mathbf{P}$ values \\
\hline 7. & Length of stay (discharged) & -2.77 & -3.45 to 2.10 & $<0.001$ \\
\hline 8. & Length of stay (if severe complication) & -1.89 & -8.42 to 4.63 & 0.57 \\
\hline 9. & Length of stay (no severe complication) & -2.58 & -3.12 to 2.04 & $<0.001$ \\
\hline 10. & Length of stay (died) & -1.33 & -5.14 to 2.48 & 0.49 \\
\hline 11. & Length of stay (discharged+died) & -2.88 & -3.42 to 2.35 & $<0.001$ \\
\hline
\end{tabular}

*Models adjusted for age, sex, premorbid function, stroke type, stroke severity and history of atrial fibrillation.

†A complication considered incapacitating, life threatening and one that prolongs hospital admission, for example, pneumonia, falls, fever, urinary tract infection, seizures, deep vein thrombosis.

aOR, adjusted OR.

spent in an SU, spending at least $90 \%$ of admission in an SU was associated with fewer severe complications than spending less than $50 \%$ of admission in an $\mathrm{SU}(\mathrm{p} \leq 0.001$; online supplementary table F).

\section{Organisational characteristics}

Hospitals with onsite neurosurgery services, located in metropolitan areas or those that were private less often kept their patients in the SU for at least $90 \%$ of their admission (table 1, online supplementary table B). Features of hospitals that were able to provide access to the SU for at least $90 \%$ of the patient's admission included those with: at least 10 beds in an SU, an SU coordinator, access to early supported discharge team, a stroke specialist research nurse involved in treatment and those in which the stroke team was involved in quality improvement in the previous 2 years (table 1 ).

\section{Factors associated with spending at least $90 \%$ of admission in an SU}

In multivariable analysis, similar factors remained relevant for likelihood of spending at least $90 \%$ of admission in an SU (table 3). For instance, patients who were admitted to an SU within 3 hours of arrival to the ED were three times more likely to spend at least $90 \%$ of their admission in an SU compared with those who were admitted after 3 hours of arrival to the ED (table 3). This finding was also similar for patients admitted in an SU within 24 hours of arrival to the ED (aOR 26.17, 95\% CI 17.08 to 40.09). Patients who were admitted to a hospital with at least 10 beds on the SU were more likely to spend at least $90 \%$ of admission in an SU compared with those admitted to a hospital with less than five beds on the SU.

\section{DISCUSSION}

To our knowledge, this is the first study to describe whether the recommendation for patients with stroke to spend at least $90 \%$ of their admission in an SU is a relevant indicator of high-quality stroke care. We demonstrated an association between patients who spent at least $90 \%$ of their admission in an SU and a reduced LOS, fewer severe complications and less discharges to a residential aged care facility. Similar results were evident from the primary analyses using the whole sample and propensity score matching, leading to more confidence in the validity of results. While results are based on stroke care provided in Australian hospitals, these findings are important for promoting and ensuring that patients with stroke spend most of their acute hospital stay in an SU and can be generalised to other countries with similar models of stroke care.

Although researchers have established that management of patients in an $\mathrm{SU}$ is associated with a reduction in length of hospital stay compared with other wards, ${ }^{2122}$ our findings have further demonstrated that length of time spent in an SU may also be important. Given the demands for beds in SUs, ${ }^{22}$ the 2-day reduction in LOS observed in our study is clinically important. Additionally, from an economic perspective, this reduction in LOS translates to potentially large cost savings. ${ }^{23} \mathrm{We}$ acknowledge that given the study design we cannot make inferences about causality. Clinically, a longer LOS may be a consequence of experiencing a severe complication (as by definition may increase time in hospital), a more severe form of stroke, or delays in access to the next stage of care. Although more patients with a severe complication were not treated in an SU, for those who did access SU care and experienced a 
Table 3 Factors associated with patients with stroke spending at least $90 \%$ of their admission in an SU

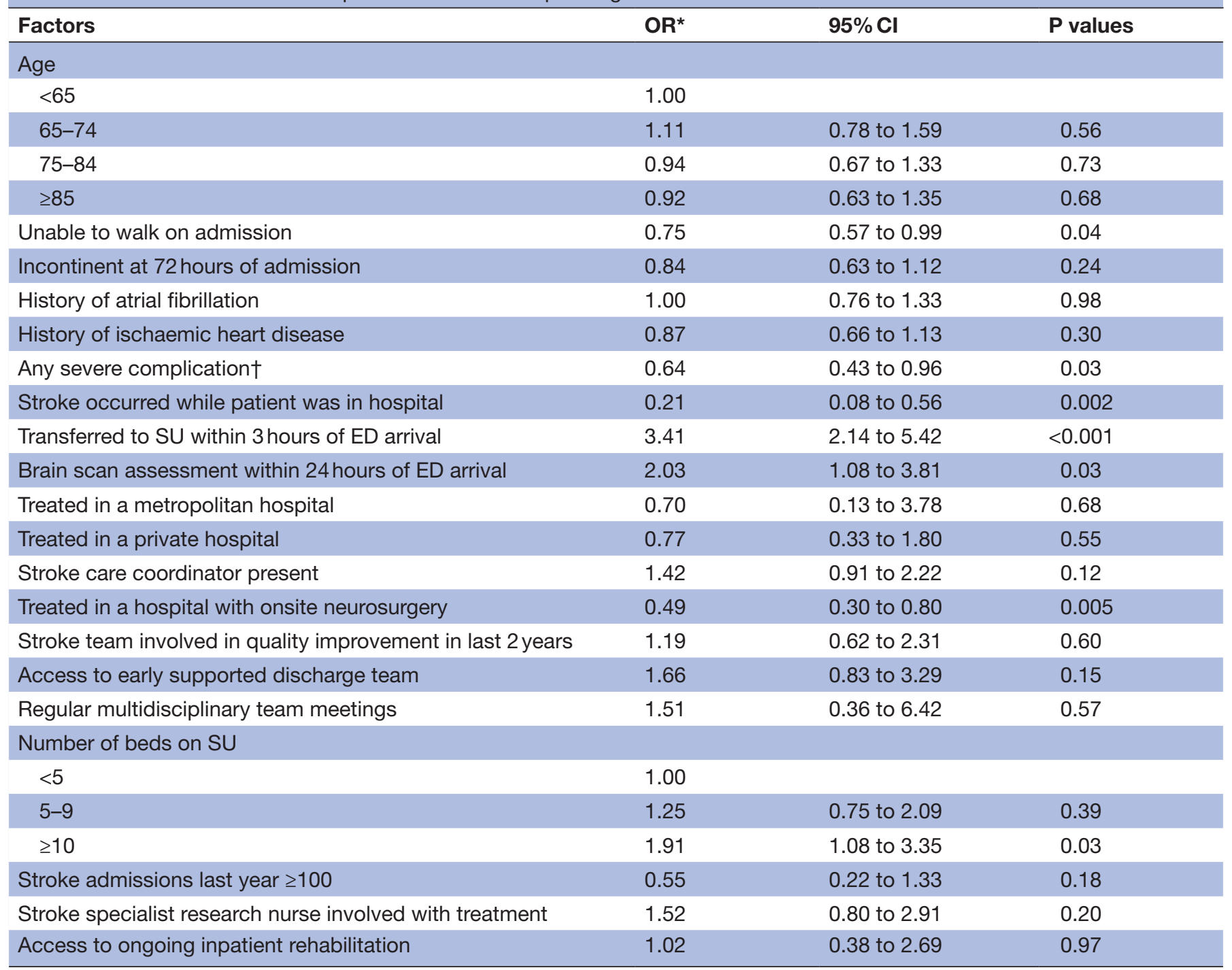

*Multivariable model adjusted for all factors listed in table; level was hospital.

†A complication considered incapacitating, life threatening and one that prolongs hospital admission, for example, pneumonia, falls, fever, urinary tract infection, seizures, deep vein thrombosis.

ED, emergency department; SU, stroke unit.

severe complication, there was no difference in LOS based on the proportion of time spent in the SU. The reduced likelihood of discharge to residential aged care facility for those spending $>90 \%$ of time in the $\mathrm{SU}$ is potentially resultant from transfers to other wards when waiting for longer term care. Regardless, with the additional trend towards reduced mortality for patients who spent at least $90 \%$ of admission in an SU, these results lend further support for ensuring that all patients with stroke spend most of their acute admission in an SU.

Given that spending at least $90 \%$ of admission in an SU potentially influences outcomes, we have further demonstrated factors that are responsible for achieving this indicator. The main finding is that being admitted to an SU within 3 hours of arrival to the ED was independently associated with spending at least $90 \%$ of admission in an SU. This finding is of great importance because early admission to an SU has also been associated with better recovery. ${ }^{24}$ Given evidence that SU care significantly reduces death and disability after stroke, ${ }^{34}$ and that the clinical guidelines for management of stroke recommend direct or early admission to an $\mathrm{SU},{ }^{5}$ our finding provides further evidence that early admission on an SU should be a high priority for clinicians and health administrators. While direct access to CT from ambulance arrival has been achieved in some hospitals with the introduction of 'Code Stroke', ${ }^{25}$ consideration of the added benefits for patients of direct admissions to SUs is warranted. Unfortunately, overall access to SU in different countries remains highly variable. For example, in Australia only $67 \%$ of the patients with stroke received SU care in $2015 .^{12}$ This is a major difference to countries like the UK where $96 \%$ of patients received SU care. ${ }^{13}$ There is 
need to improve access as well as timely admission to an SU.

Additionally, having a brain scan within 24 hours of arrival to the ED was associated with spending at least $90 \%$ of admission in an SU. An early brain scan is important for confirming the type of stroke and to exclude stroke mimics, thus enabling commencement of time-dependent therapies. ${ }^{5}$ The fact that patients who spent at least $90 \%$ of their admission in an SU were more likely to begin rehabilitation therapy within 48 hours of initial assessment highlights the importance of this indicator. These findings provide the impetus for early assessment and early admission of all patients with stroke onto an $\mathrm{SU}$ as this may help to advocate for patients to spend most of their acute hospital stay in an SU.

Having at least 10 beds on the SU was associated with spending at least $90 \%$ of admission in an SU and this finding provides a strong argument for capacity building and potential redistribution of resources within hospitals to better support care for patients with stroke where there is the relevant throughput of patients. $^{12}$

There are some limitations that must be acknowledged. The time for discharge from the SU and hospital was unavailable. Therefore, our analysis was limited to dates which do not provide fine granularity that time would have provided. Also, some observations were excluded because of invalid or missing dates. The comprehensive dataset did allow us to adjust our multivariable models for a number of comorbidities and patient variables, including stroke severity, for which we used a validated prognostic model. However, we acknowledge that the influence of unmeasured confounders such as socioeconomic status and other comorbidities could not be fully addressed. Data on patients' ward of first admission or transfers during the admission were not collected which precludes us from making definitive conclusions such as whether individuals with severe stroke or who suffer severe complications are admitted or transferred to the intensive care unit or other high-dependency units first before admission on an SU or during their acute stay. Although there is evidence that $\mathrm{SU}$ care reduces mortality through prevention and treatment of infection and immobility-related complications, ${ }^{26}$ having these additional data would have provided insight to why patients with severe stroke or severe complications were less likely to spend at least $90 \%$ of their admission in an SU. Additional longer term outcomes would also be beneficial. Given these limitations and the nature of the study design which precludes us from drawing firm conclusions about temporal relationships, these findings should be interpreted with caution. The above limitations notwithstanding, a strength of our study is the large data set from a wide cross-section of Australian hospitals which provides national representation.

\section{CONCLUSIONS}

Spending at least $90 \%$ of time in an SU is a useful measure of care quality and was associated with better patient outcomes such as shorter LOS, fewer severe complications and less discharge to aged care facilities. Our findings have important implications for clinical practice and development of new models of stroke care.

\section{Author affiliations}

${ }^{1}$ Translational Public Health and Evaluation Division, Stroke and Ageing Research, School of Clinical Sciences at Monash Health, Monash University, Clayton, Victoria, Australia

${ }^{2}$ Stroke Division, The Florey Institute of Neuroscience and Mental Health, University of Melbourne, Heidelberg, Victoria, Australia

${ }^{3}$ Nursing Research Institute, St Vincent's Health Australia (Sydney) and Australian Catholic University, Darlinghurst, New South Wales, Australia

${ }^{4}$ Department of Medicine and Neurology, Royal Melbourne Hospital, University of Melbourne, Parkville, Victoria, Australia

Acknowledgements We acknowledge the Stroke Foundation for coordinating this National Stroke Audit and recognise the AuSDaT tool used to collect these data. We gratefully acknowledge the hospitals participating in the National Stroke Audit and all the clinicians who contributed to data collection over the audit cycles. Preliminary results from this study were presented at the 27th Annual Scientific Meeting of the Stroke Society of Australasia, Queenstown, 2017 and have been published http://journals.sagepub.com/doi/10.1177/1747493017720548.

Contributors DB, MFK and DAC were responsible for study concept and design. $\mathrm{DB}, \mathrm{TP}, \mathrm{JK}$ and MFK were responsible for statistical analyses. DB drafted the manuscript. DB, MFK, TP, JK, SM, BCVC and DAC interpreted the data, critically revised the manuscript for important intellectual context and approved the final version for submission. DB, MFK, TP, JK, SM, BCVC and DAC agreed to be accountable for all aspects of the work.

Funding The following authors receive research fellowship support from the NHMRC: DAC (1063761-cofunded Heart Foundation), MFK (Early Carer Fellowship 1109426).

Competing interests BCVC and SM are members of the Stroke Foundation Clinical Council.

\section{Patient consent Not required.}

Ethics approval Ethics approval was granted through Monash University Human Research Ethics Committee (CF16/825-2016000402).

Provenance and peer review Not commissioned; externally peer reviewed.

Data sharing statement Contact can be made with the corresponding author for queries relating to unpublished data.

Open access This is an open access article distributed in accordance with the Creative Commons Attribution Non Commercial (CC BY-NC 4.0) license, which permits others to distribute, remix, adapt, build upon this work non-commercially, and license their derivative works on different terms, provided the original work is properly cited, appropriate credit is given, any changes made indicated, and the use is non-commercial. See: http://creativecommons.org/licenses/by-nc/4.0/.

\section{REFERENCES}

1. Feigin VL, Krishnamurthi RV, Parmar P, et al. Update on the Global Burden of Ischemic and Hemorrhagic Stroke in 1990-2013: The GBD 2013 Study. Neuroepidemiology 2015;45:161-76.

2. Cadilhac DA, Pearce DC, Levi CR, et al. Improvements in the quality of care and health outcomes with new stroke care units following implementation of a clinician-led, health system redesign programme in New South Wales, Australia. Qual Saf Health Care 2008;17:329-33.

3. Stroke Unit Trialists' Collaboration. Organised inpatient (stroke unit) care for stroke. Cochrane Database Syst Rev 2013:CD000197.

4. Cadilhac DA, Andrew NE, Lannin NA, et al. Quality of acute care and long-term quality of life and survival: The Australian Stroke Clinical Registry. Stroke 2017;48:1026-32.

5. National Stroke Foundation. Clinical guidelines for stroke management 2010. Melbourne: National Stroke Foundation, 2010. 
6. Intercollegiate Stroke Working Party. The national clinical guidelines for stroke. London: Royal College of Physicians of London, 2016.

7. Stroke Foundation. National stroke audit acute services report 2017. Melbourne: Stroke Foundation, 2017.

8. Purvis T, Moss K, Francis L, et al. Benefits of clinical facilitators on improving stroke care in acute hospitals: a new programme for Australia. Intern Med J 2017;47:775-84.

9. National Health Service Improvement. Implementing best practice in acute care: case studies from the Stroke Improvement Programme projects. Leicester: National Health Service, 2010.

10. Middleton S, Levi C, Dale S, et al. Triage, treatment and transfer of patients with stroke in emergency department trial (the $\mathrm{T}^{3}$ Trial): a cluster randomised trial protocol. Implement Sci 2016;11:139.

11. Alonso A, Ebert AD, Kern R, et al. Outcome Predictors of Acute Stroke Patients in Need of Intensive Care Treatment. Cerebrovasc Dis 2015;40:10-17.

12. National Stroke Foundation. National stroke audit - acute services report 2015. Melbourne: National Stroke Foundation, 2015

13. Royal College of Physicians. Sentinel Stroke National Audit Programme (SSNAP) Clinical Audit, Public Report. London: Royal College of Physicians, 2016.

14. Australian Commission on Safety and Quality in Health Care. Acute stroke clinical care standard. Sydney: ACSQHC, 2015.

15. Rudd AG, Hoffman A, Irwin P, et al. Stroke unit care and outcome: results from the 2001 National Sentinel Audit of Stroke (England, Wales, and Northern Ireland). Stroke 2005;36:103-6.

16. von Elm E, Altman DG, Egger M, et al. The Strengthening the Reporting of Observational Studies in Epidemiology (STROBE) statement: guidelines for reporting observational studies. PLoS Med 2007; $4: \mathrm{e} 296$.
17. Cadilhac D, Lannin N, Anderson C, et al. The Australian Stroke Clinical Registry Annual Report 2016. Melbourne: The Florey Institute of Neuroscience and Mental Health, 2017.

18. Harris D, Cadilhac D, Hankey GJ, et al. National Stroke Audit: The Australian Experience. Clin Audit 2010;2:25-31.

19. Counsell C, Dennis M, McDowall M, et al. Predicting outcome after acute and subacute stroke: development and validation of new prognostic models. Stroke 2002;33:1041-7.

20. Sim J, Teece L, Dennis MS, et al. Validation and Recalibration of Two Multivariable Prognostic Models for Survival and Independence in Acute Stroke. PLoS One 2016;11:e0153527.

21. Zhu HF, Newcommon NN, Cooper ME, et al. Impact of a stroke unit on length of hospital stay and in-hospital case fatality. Stroke 2009;40:18-23.

22. Cadilhac DA, Purvis T, Kilkenny MF, et al. Evaluation of rural stroke services: does implementation of coordinators and pathways improve care in rural hospitals? Stroke 2013;44:2848-53.

23. Phillips SJ, Eskes GA, Gubitz GJ. Queen Elizabeth II Health Sciences Centre Acute Stroke Team. Description and evaluation of an acute stroke unit. CMAJ 2002;167:655-60.

24. Silvestrelli G, Parnetti L, Paciaroni M, et al. Early admission to stroke unit influences clinical outcome. Eur J Neurol 2006;13:250-5.

25. Gomez CR, Malkoff MD, Sauer CM, et al. Code stroke. An attempt to shorten inhospital therapeutic delays. Stroke 1994;25:1920-3.

26. Govan L, Langhorne P, Weir CJ. Stroke Unit Trialists Collaboration. Does the prevention of complications explain the survival benefit of organized inpatient (stroke unit) care?: further analysis of a systematic review. Stroke 2007;38:2536-40. 